\title{
New Developments and Future Perspectives of Gaseous Detectors
}

\author{
Maxim Titov ${ }^{\mathrm{a}, \mathrm{b}}$ \\ ${ }^{\text {a } C E A / S A C L A Y, ~ D A P N I A, ~} 91191$ Gif-sur-Yvette, France \\ ${ }^{\mathrm{b}}$ Albert-Ludwigs University of Freiburg, Physics Institute, Freiburg, Germany
}

\begin{abstract}
Gaseous detectors are fundamental components of all present and planned high energy physics experiments. Over the past decade two representatives (GEM, Micromegas) of the Micro-Pattern Gas Detector (MPGD) concept have become increasingly important; the high radiation resistance and excellent spatial and time resolution make them an invaluable tool to confront future detector challenges at the next generation of colliders. Novel structures where GEM and Micromegas are directly coupled to the CMOS multi-pixel readout represent an exciting field and allow to reconstruct fine-granularity, two-dimensional images of physics events. Originally developed for the high energy physics, MPGD applications have expanded to astrophysics, neutrino physics, neutron detection and medical imaging.
\end{abstract}

\section{Introduction}

The compelling scientific goals of future high energy physics experiments are a driving factor in the development of advanced detector technologies. With many fundamental issues within the experimental reach at the Large Hadron Collider (LHC), a large $R \& D$ effort was devoted to the optimization of existing devices and the development of innovative concepts for radiation detection. All four major LHC experiments (ATLAS, CMS, ALICE and LHCb) will extensively use gas detectors, depending on the need for trigger and bunch crossing tagging (GEM, Resistive Plate Chambers, Thin Gap Chambers), high-rate tracking and particle identification (Straw-Type Detectors, Time Projection Chamber, Drift Tubes, Cathode Strip Chambers) and single photon detection (Multi-Wire Proportional Chamber with CsI photocathodes). In future colliders, gaseous detectors are planned to be used at the ten times higher luminosity upgrade of LHC (the so-called superLHC or SLHC) or at the foreseen International Linear Collider (ILC). The related $R \& D$ challenges come on one 
hand from the development of radiation-hard detector concepts and high granularity readout and on the other hand from the need for ultra-high-precision tracking with minimum material budget.

Within the broad family of different gas detector technologies, two innovative MPGD concepts, GEM and Micromegas, have now reached maturity and play a prominent role in modern HEP experiments. Their attractive properties such as excellent spatial resolution, fast signal response, low radiation length and high rate capability combined with radiation hardness, motivate their use at the future SLHC and ILC colliders. There are many common issues to be addressed at the next generation of $e^{+} e^{-}$and $p p$ machines, necessitating more fundamental $R \& D$ in the field of micro-pattern devices, which would be beneficial for both communities. This paper reviews recent advances of GEM and Micromegas detectors, with a focus on design principles, performance, and operational experience, and discusses the most promising directions in future developments and applications.

\section{Gaseous detectors at the LHC: System Aspects of Modern Ex- periments}

The development of modern LHC detectors presented formidable challenges, which are at the same time of great technological, engineering and organizational complexity. Typical time-scales have stretched over 20 years starting from the concept, progressing through intensive $R \& D$, design and prototyping, mass production, installation and finally system integration and commissioning. These detectors are not just bigger versions of currently running experiments; several new issues had to be addressed during their construction:

- Radiation hardness at extremely high radiation levels;

- Intrinsic performance of innovative detector technologies integrated into large systems at the physics frontier;

- A coherent and system oriented approach from the $R \& D$ phase to commissioning, including many-year-long strong partnership with industry;

- Sub-detector systems become so large and complex that their construction has to be shared around the world.

The LHC gaseous detectors will not be reviewed in this paper; they are covered in a number of dedicated articles [3,4]. Although achieving the intrinsic performance in the lab captures most of experimenter's attention, the complexity of the overall system approach remains the area where most systems experience major setbacks. Understanding of all system aspects should be a focus of vigorous $R \& D$ to develop common solutions for upgraded and new experiments. The commissioning of the LHC experiments is about to reach 
a successful completion over the coming year. Using the ATLAS Muon Spectrometer as an example of a modern gaseous detector, the main challenges of achieving the intrinsic performance in a large system using standard wire chamber technology are discussed.

\subsection{ATLAS Muon Detector: Modern Large-Volume Spectrometer}

The ATLAS Muon Spectrometer has been designed for standalone tracking with a momentum resolution of $2.5 \%$ for transverse momenta up to $250 \mathrm{GeV}$ (limited by multiple scattering) and better than $10 \%$ up to $1 \mathrm{TeV}$ (limited by the muon chamber measurement accuracy) [1]. A total of 370000 Monitored Drift Tubes (MDT), arranged in 1200 chambers and covering an active area of more than $5500 \mathrm{~m}^{2}$, have been built at 13 construction sites worldwide over a period of 5 years [2]. The basic detection element of the ATLAS MDT is an aluminum tube of $3 \mathrm{~cm}$ diameter and $500 \mu \mathrm{m}$ wall thickness, with a $50 \mu \mathrm{m}$ diameter central gold-plated $\mathrm{W}$-Re wire. The detector is operated with a low longitudinal diffusion $\mathrm{Ar} / \mathrm{CO}_{2}$ (93:7) mixture at 3 bar absolute pressure. Despite various improvements, position-sensitive detectors based on wire structures are limited by basic diffusion processes and space charge effects in the gas to localization accuracies around $100 \mu \mathrm{m}$. The presence of slow-moving ions from electron avalanches generates a positive space charge in the drift tube, which modifies the electric field and leads to an uncertainty in the space-to-drift-time relation. This results in a degradation of the singletube resolution with increasing irradiation rate, especially for large impact radii (see Fig. 1a). Close to the sense wire the space charge effects lower the electric field causing a gain drop.

a)

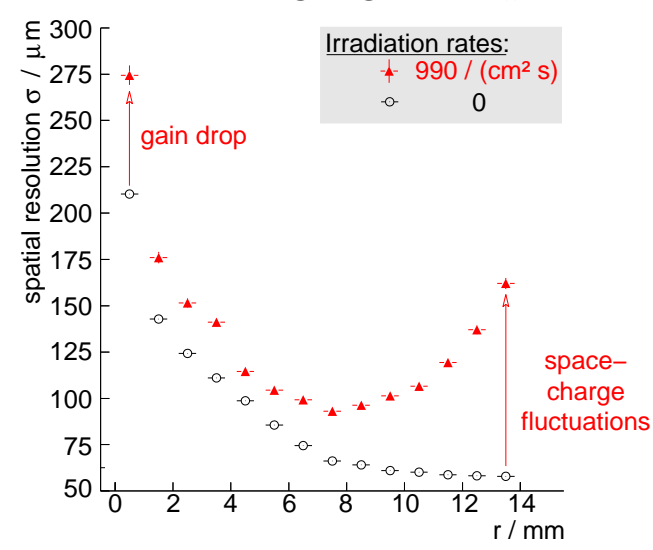

b)

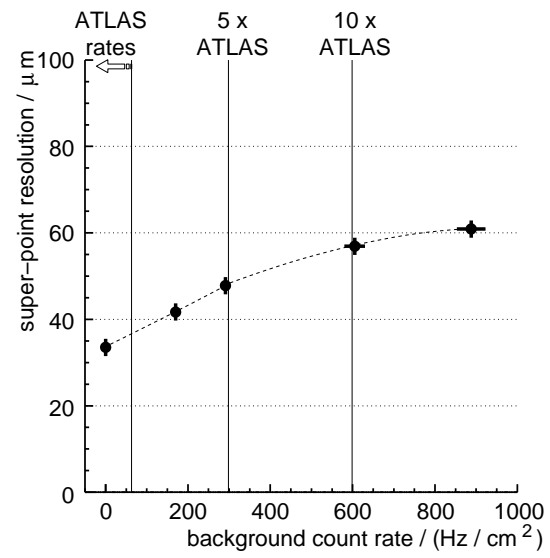

Fig. 1. a) Single tube resolution as a function of the muon impact radius, with and without irradiation. b) Position resolution of the MDT chamber as a function of the background particle flux in the ATLAS Muon Spectrometer.

The momentum resolution goals in the ATLAS Muon Spectrometer imply an overall precision of $\sim 50 \mu \mathrm{m}$ on the track points, given the available bending power. To improve the resolution of a chamber beyond the single wire limit, 
the MDT chambers are constructed from $2 \times 3$ monolayers of drift tubes, glued on either side of a rigid support structure. The high accuracy of the standalone measurement puts stringent demands on the mechanical precision of the sense wire location $(20 \mu \mathrm{m})$. In addition, an internal alignment system with 12000 optical sensors has to monitor continuously the deformations and movements of the precision chambers with a relative accuracy of $30 \mu \mathrm{m}$. The space-drifttime relation for a single tube has to be known with an accuracy of $20 \mu \mathrm{m}$. Finally, the inhomogeneity of the magnetic field throughout the whole detector volume must be measured and monitored to an accuracy of approximately $20 \mathrm{G}$. Recent studies have demonstrated that it is feasible to reach the required system performance, as shown in Fig. 1b. A spatial resolution of $\sigma<60 \mu m$ per MDT chamber was measured at particle fluxes of up to $600 \mathrm{~Hz} / \mathrm{cm}^{2}$, which corresponds to 10 times the highest background rate expected in the ATLAS Muon Spectrometer.

\section{Micro-Pattern Gaseous Detectors}

Modern photo-lithographic technology has enabled a series of inventions of novel MPGD concepts: Micro-Strip Gas Chamber (MSGC), GEM, Micromegas and many others [5], revolutionizing cell size limits for many gas detector applications. The MSGC, a concept invented in 1988 by A. Oed [6], was the first of the microstructure gas detectors. Consisting of a set of tiny metal strips laid on a thin insulating substrate, and alternatively connected as anodes and cathodes, the MSGC turned out to be easily damaged by discharges induced by heavily ionizing particles and destroying the fragile electrode structure [7]. The more powerful GEM and Micromegas concepts fulfill the needs of high-luminosity colliders with increased reliability in harsh radiation environments. By using smaller feature size compared to classical gas counters, these detectors offer intrinsic high rate capability (fine pitch and fast collection of positive ions) [8,9], excellent spatial resolution $(\sim 30 \mu m)[10,11]$, and single-photoelectron time resolution in the nanosecond range $[12,13]$.

a)

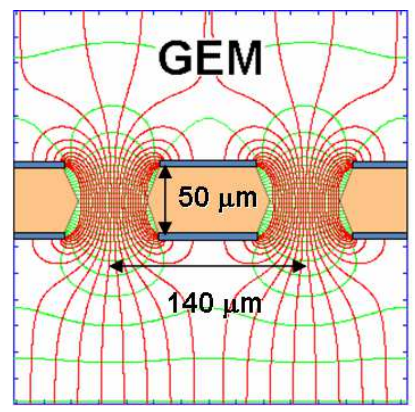

b)

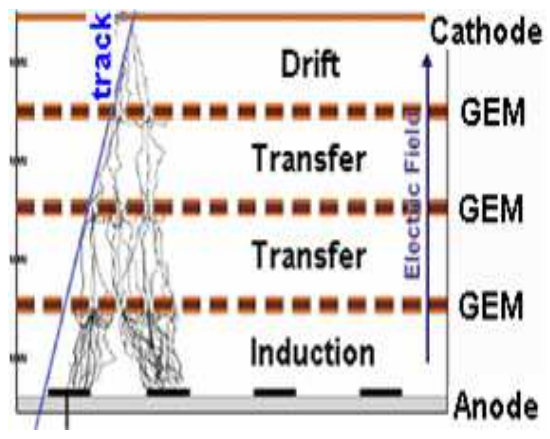

Fig. 2. a) Schematics and electric field map of the GEM amplification cell. b) Schematical drawing of the triple-GEM detector. 
Introduced in 1996 by F. Sauli [14], a GEM consists of a set of holes, arranged in a hexagonal pattern (typically $70 \mu \mathrm{m}$ diameter at $140 \mu \mathrm{m}$ pitch), chemically etched through copper-kapton-copper thin-foil composite. Application of a potential difference between the two sides of the GEM generates the field map shown in Fig. 2a: electrons released by the ionization in the gas drift into the holes and multiply in the high electric field $(50-70 \mathrm{kV} / \mathrm{cm})$. Sharing the avalanche multiplication among several cascaded electrodes (see Fig. 2b) allows to operate triple-GEM detectors at overall gains above $10^{4}$ in the presence of highly ionizing particles while eliminating the risk of hazardous discharges $\left(<10^{-12}\right.$ per hadron). This is the major advantage of the GEM technology $[15,16]$. A unique property of the GEM detector is the complete decoupling of the amplification stage (GEM) and the readout electrode (PCB), which operates at unity gain and serves only as a charge collector. This offers some freedom in the optimization of the anode readout structure, which can be made of pads or strips of arbitrary pattern [17]. GEMs can also be easily bent to form cylindrically curved ultra-light detectors, as preferred for inner tracker applications [18,19]. Controlled etching of GEM foils (decreasing the thickness of the copper layer from 5 to $1 \mu \mathrm{m}$ ) allows to reduce the material budget in triple GEMs to $1.5 \times 10^{-3} X_{0}$, which is about one half of a 300- $\mu m$-thick Si-microstrip detector [20]. Recently, several companies (Tech Etch and 3M) have started to develop an industrial version of GEMs [21]. Their mechanical and physical properties are found to be similar to the foils produced at the CERN workshop [22].

a)

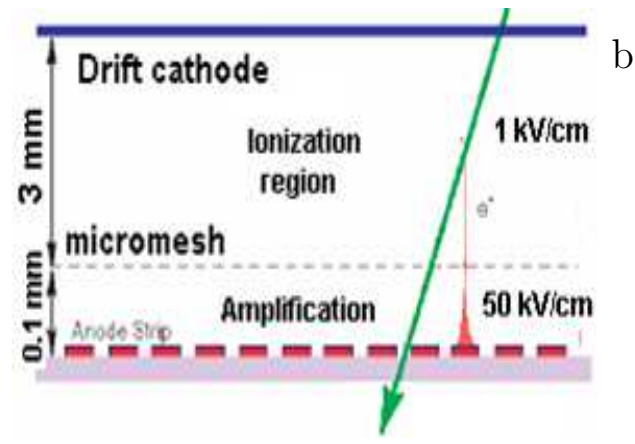

b)

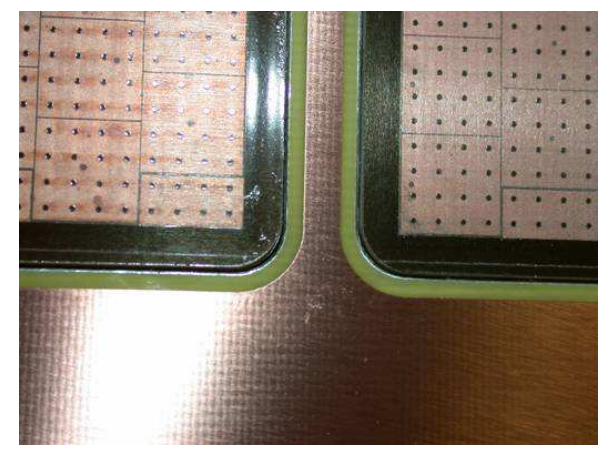

Fig. 3. a) Schematical drawing of the Micromegas detector. b) Photograph of the "Bulk" Micromegas detectors. Pillars of $400 \mu \mathrm{m}$ diameter every $2 \mathrm{~mm}$ are visible.

Introduced in 1996 by I. Giomataris [23], a micro-mesh gaseous structure (Micromegas) is a double stage parallel plate avalanche counter (see Fig. 3a). It consists of a few mm conversion region (electric field $\sim 1 \mathrm{kV} / \mathrm{cm}$ ) and a narrow multiplication gap (25-150 $\mu \mathrm{m}, 50-70 \mathrm{kV} / \mathrm{cm})$, located between a thin metal grid (micromesh) and the readout electrode (strips/pads of conductor printed on an insulator board). To preserve a distance between the anode and the grid mesh, spacers made of insulating material are used. The small amplification gap is a key element in Micromegas operation and gives rise to excellent spatial resolution: $12 \mu \mathrm{m}$ accuracy (limited by the pitch of micromesh) is achieved for MIPs with a strip pitch of $100 \mu \mathrm{m}$ and low diffusion $C F_{4} / i C_{4} H_{10}$ 
(80:20) mixture [11]. Micromegas exploits the saturation characteristics of the Townsend coefficient at high fields to achieve a reduced dependence of the gas gain on gap variations, leading to very good energy resolution $(\sim 12 \%$ FWHM at $6 \mathrm{keV})[24]$.

A big step in the direction of the industrial manufacturing of large-size detectors is the development of the "Bulk" Micromegas technology [25]. The basic idea is to built the whole detector in a single process: the anode plane with copper strips, a photo-imageable polyamid film and the woven mesh are laminated together at high temperature forming a single object. At the end, the micromesh is sandwiched between 2 layers of insulating material, which is removed after UV exposure and chemical development. Several large "Bulk" Micromegas $\left(27^{*} 26 \mathrm{~cm}^{2}\right)$, as prototypes for T2K/TPC, have been produced (see Fig. 3b) and successfully tested in the HARP TPC field cage at CERN inside a magnetic field $[26,27]$. This technique has been recently extended to build even larger-size detectors, up to $65 \times 55 \mathrm{~cm}^{2}$, in a single piece [28].

\subsection{High-Rate Tracking and Triggering}

COMPASS is a first high-luminosity experiment at CERN which pioneered the use of GEM and Micromegas detectors for high-rate particle tracking, reaching $25 \mathrm{kHz} / \mathrm{mm}^{2}$ in the near-beam area. Both technologies have achieved a tracking efficiency of close to $100 \%$, a spatial resolution of the order of 70 - $100 \mu \mathrm{m}$ and a time resolution of $\sim 10 \mathrm{~ns}$ [29], [30]. The excellent performance and radiation hardness of 22 large-size triple-GEM $\left(30^{*} 30 \mathrm{~cm}^{2}\right)$ and 12 Micromegas $\left(40 * 40 \mathrm{~cm}^{2}\right)$ detectors after several years of successful operation has demonstrated the large-scale reliability and robustness of the MPGD concept. No degradation of performance is observed in COMPASS detectors after an accumulated charge of a few milliCoulombs $/ \mathrm{mm}^{2}$, corresponding to an equivalent flux of $\sim 10^{11} \mathrm{MIPs} / \mathrm{mm}^{2}$. For the COMPASS physics program in 2007, a set of triple-GEM trackers with pixel readout $\left(1 \times 1 \mathrm{~mm}^{2}\right)$ in the central region and 2D strip readout in the periphery is being built [31].

The intrinsic time resolution of GEM and Micromegas detectors is determined by the time distribution of the primary ionization clusters and the signal amplitude fluctuations, both in the ionization and multiplication processes. A time resolution of $\sim 10$ ns (RMS) measured in the COMPASS GEM detectors is limited by the moderate electron drift velocity in $\mathrm{Ar} / \mathrm{CO}_{2}$ and by the readout electronics. Using fast $C F_{4}$-based mixtures, a time resolution of about $5 \mathrm{~ns}$ (RMS) [32] and an efficiency of $96 \%$ in a 20 ns time window can be achieved [33], adequate to resolve two bunch crossings at the high luminosity colliders. GEMs have entered the LHC program; they will be used for triggering in the LHCb Muon System [33] and in the TOTEM Telescopes [18,34]. 


\subsection{Time Projection Chamber Readout}

The Time Projection Chamber (TPC) concept, invented in 1976 [35], has been the prime choice for large tracking systems in $e^{+} e^{-}$colliders (PEP-4, ALEPH, DELPHI) and proved its unique resolving power in heavy-ion collisions (NA35, NA49 and STAR). It is an ideal tracker with minimal material budget before the calorimeters for high-multiplicity topologies occurring at low rates. A TPC consists of a large gas volume, with a uniform electric field applied between the central electrode and a grid at the opposite side. The ionization trails produced by charged particles drift towards the readout end-plate where a 2D image of tracks is reconstructed; the third coordinate is measured using the drift time information. The large number of 3D space points makes for a robust and efficient tracking system, even for tracks within the densely collimated jets at the future ILC, and offers the capability to measure particle momenta and to perform particle identification through $\mathrm{dE} / \mathrm{dx}$ measurements. A conventional readout structure, based on MWPC and pads, is a benchmark for the "most modern" ALICE TPC, designed to cope with extreme instantaneous particle densities produced in heavy-ion collisions at the LHC. This detector incorporates innovative and state of the art technologies, from the mechanical structures to the readout electronics and data processing chain [36,37]. To limit distortions of the ALICE TPC intrinsic spatial resolution $\left(\sigma_{r \phi} \sim 1000 \mu \mathrm{m}\right.$ for $250 \mathrm{~cm}$ drift length), the temperature gradient in an $88 \mathrm{~m}^{3}$ gas volume space must not exceed $0.1^{\circ} \mathrm{C}[38]$.

a)

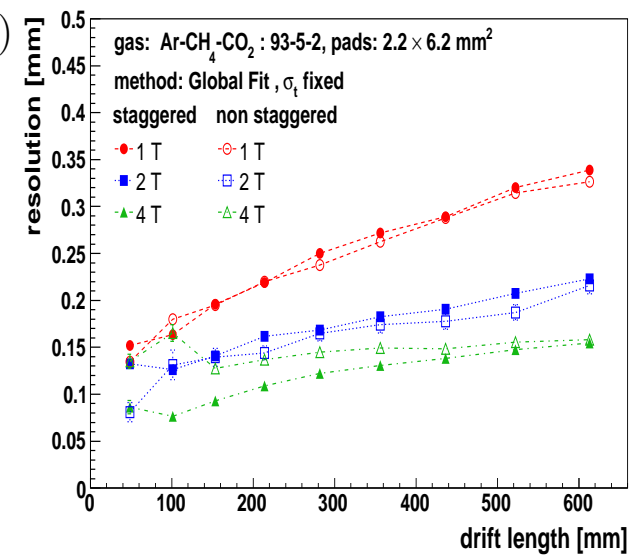

b)

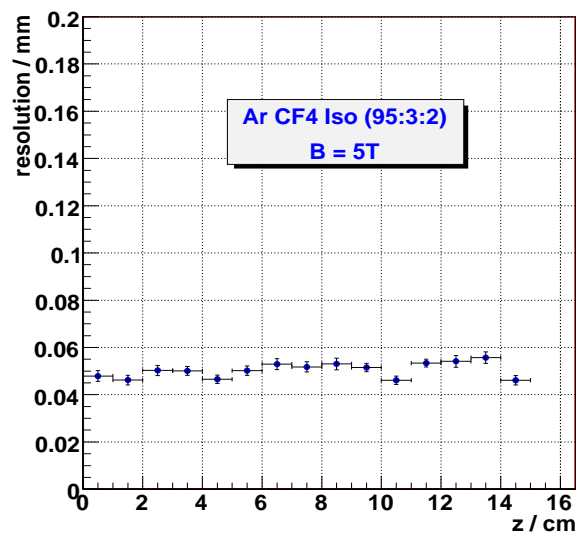

Fig. 4. Transverse spatial resolution as a function of drift distance for $2 \times 6 \mathrm{~mm}^{2}$ pads: a) GEM-TPC with the $\mathrm{Ar} / \mathrm{CH}_{4} / \mathrm{CO}_{2}(93: 5: 3)$ mixture at $1 \mathrm{~T}, 2 \mathrm{~T}$ and $4 \mathrm{~T}$ fields; b) Micromegas-TPC, using charge dispersive readout, with $\mathrm{Ar} / \mathrm{CF}_{4} / i \mathrm{C}_{4} \mathrm{H}_{10}(95: 3: 2)$ mixture in $5 \mathrm{~T}$ field.

$R \& D$ for a high-precision TPC is ongoing in the international ILC-TPC collaboration [39]. The main topics are the construction of a low material-budget field cage and the development of gas amplification end-plates using GEM or Micromegas. In the context of the particle flow concept at the ILC, the requirements for charged particle tracking are mainly high efficiency and double 
track resolution. In addition, a momentum resolution of $\sigma\left(1 / p_{t}\right) \approx 10^{-4} / \mathrm{GeV}$ in the TPC alone has to be achieved to reconstruct Higgs mass in a model independent way (exploiting the recoil mass technique) in Higgs-strahlung events $\left(e^{+} e^{-} \rightarrow Z H \rightarrow l l X\right)$ and to measure the end-point momentum in supersymmetric slepton decays $\left(\tilde{l} \tilde{l} \rightarrow \tilde{\chi}_{1}^{0} \tilde{\chi}_{1}^{0} l l\right)$. Therefore, at least 200 space points with an average transverse resolution of $100 \mu \mathrm{m}$ have to be measured over a maximum drift length of $250 \mathrm{~cm}$ in a $4 \mathrm{~T}$ field, and a multi-track separation of $1 \mathrm{~mm}$ has to be reached. These requirements are beyond the limits of MWPC but can be fulfilled with GEM or Micromegas readout, which offer a number of advantages: negligible $\mathrm{E} \times \mathrm{B}$ track distortion effects, narrow pad response function (PRF) and intrinsic ion feedback suppression.

The principle of the ILC-TPC MPGD concept has been successfully validated over the last years. The single point resolution of $\sim 100 \mu \mathrm{m}$ has been achieved with a GEM-TPC prototype after $60 \mathrm{~cm}$ of drift in a $4 \mathrm{~T}$ field (see Fig. 4a). The spatial resolution for low magnetic fields shows the expected dependence on the drift length, which is caused by diffusion. For high fields the GEM-TPC resolution is dominated by the PRF [40]. Recent studies with a Micromegas TPC using a charge-dispersive readout technique have demonstrated an excellent single point resolution of $50 \mu \mathrm{m}$ over the $15 \mathrm{~cm}$ drift length in a $5 \mathrm{~T}$ field, as shown in Fig. 4b [41]. In this case a high-surface-resistivity thin film is laminated to the anode forming a distributed two-dimensional RC network with respect to the readout plane [42]. The arriving avalanche charge at the anode disperses with the RC system time constant (determined by the anode surface resistivity and capacitance per unit area). This avoids degradation of the point resolution due to single-pad hits at short drift distances. A fractional ion backflow into the drift volume down to $0.2 \%$ is measured both with GEM and Micromegas, which in combination with modern low-noise electronics might allow to avoid the use of a gating grid. An attractive approach to use a GEM foil for gating, if needed, has been proposed in [43]. However, the effect of electron transmission losses on the spatial resolution (at low GEM voltages used for gating) has still to be experimentally measured. Other issues to be addressed include optimization of single-point and double-track resolution in presence of background, demonstration of large-system performance in a $4 \mathrm{~T}$ field with control of systematics, and end-plate design for minimal material.

The future MPGD-TPC developments are not limited to the $R \& D$ for the linear collider: in fact, they will be used in a variety of applications. Employing the "Bulk" technology, 72 large Micromegas $\left(34 \times 36 \mathrm{~cm}^{2}\right)$ will be built for the T2K/TPC detector to instrument an area of almost $10 \mathrm{~m}^{2}$. TPCs will eventually disappear from hadron colliders because of the long memory time and proneness to space charge accumulation. However, they will prevail at future lepton and heavy-ion colliders [44] where they constitute one of the most cost-effective central tracking systems. 


\section{Pixel Readout for Micro-Pattern Gas Detectors}

Advances in the micro-electronics industry and advanced PCB technology have been very important for the development of modern gas detectors with increasingly smaller pitch size. The fine granularity and high-rate capability of micro-pattern devices can be fully exploited using a high-density pixel readout with a size corresponding to the intrinsic width of the detected avalanche charge. However, for a pixel pitch of the order of $100 \mu m$, technological constraints severely limit the maximum number of channels that can be brought to the external front-end electronics. An elegant solution is to use a CMOS pixel chip assembled directly below the GEM or Micromegas amplification structure and serving as an integrated charge collecting anode. With this arrangement avalanche electrons are collected on the top metal layer of the CMOS ASIC; every input pixel is then directly connected to the amplification, digitization and sparsification circuits integrated in the underlying active layers of the CMOS technology. Using this approach, gas detectors can reach the level of integration typical of solid-state pixel devices.

Particle detectors are designed to achieve the sensitivity required to study physics processes of interest. The multi-pixel anode readout of micro-pattern gas detectors allows a true 2D image reconstruction and opens novel detection opportunities in:

- Astronomical $X$-ray polarimetry (2-10 keV energy range);

- Position sensitive single-electron detection;

- Time Projection Chamber readout;

- High-rate particle tracking;

- Advanced Compton Telescopes (0.4-50 MeV energy range);

- Low energy nuclear recoil reconstruction (WIMP interactions).

The advent of finely segmented MPGD with pixel read-out could lead to the appearance of a highly efficient $X$-ray polarimeter in the $2-10 \mathrm{keV}$ energy band, which would allow to measure simultaneously position- and energy-resolved linear polarisation [45]. The real breakthrough was the development of an analog, low-noise and high granularity (50 $\mu \mathrm{m}$ pitch) multi-pixel ASIC [46][49] shown in Fig. 5a. So the initial direction and dynamics of photoelectron energy loss in the gas can be accurately tracked before they are distorted by Coulomb scattering.

Following a similar approach, a binary multi-pixel CMOS chip ("Medipix2"), originally developed for $X$-ray imaging [50] has been shown to work with Micromegas and GEM detectors [51]-[53]. Approximately $75 \%$ of every pixel in the Medipix2 matrix are covered with an insulating passivation layer. Hence, the avalanche electrons are collected on the conductive bump-bonding pads, 
a)

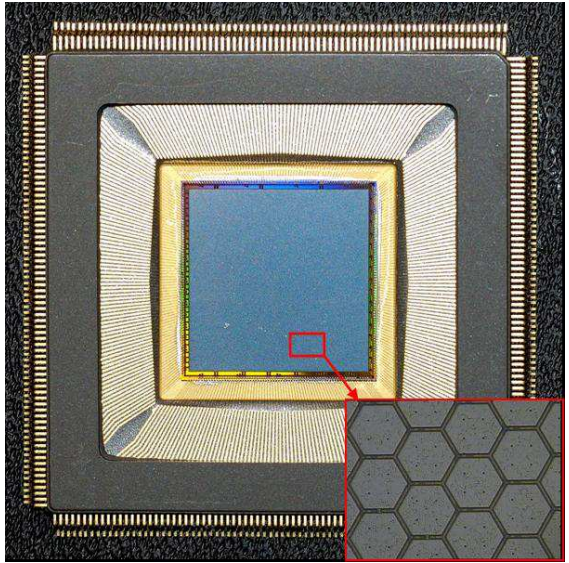

b)

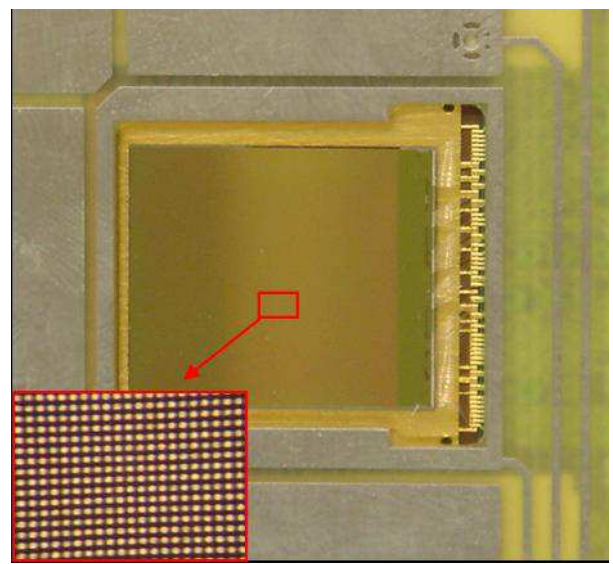

Fig. 5. a) Photo of the analog CMOS ASIC with hexagonal pixels, bonded to the ceramic package [48]. b) Photo of the Medipix2 chip [50]; the $25 \mu \mathrm{m}$ wide conductive bump bond openings used for electron collection are seen as a matrix of dots [53].

exposed to the gas (see Fig. 5b). Within the EUDET program [54], a modification of the Medipix2 chip ("TimePix" [55]) which allows to measure the drift time information of primary electrons has been designed, produced and already tested with GEM and Micromegas gas amplification systems. The Timepix chip uses an external clock up to $100 \mathrm{MHz}$ as a time reference. Each pixel in the chip matrix can be programmed to record either the electron arrival time with respect to an external shutter ("TIME" mode) or the timeover-threshold ("TOT" mode) information, providing a pulse-height measurement. Developed as a potential readout for the ILC TPC to exploit the ultimate spatial and double-track resolution, the TimePix chip can reconstruct the 3D-space points of individual electron clusters and thus count the number of ionization clusters per unit length for particle discrimination. Significant progress has also been made in the development of simulation tools and the comparison with data recorded with the Medipix2 and Timepix chips [56]. Reading out large volume TPCs with highly segmented anode planes is also a key point for high-resolution track imagers proposed for an advanced Compton Telescope $[57,58]$ and for the detection of possible signatures of elastic interactions of WIMPs $[59,60]$. The primary advantage of a pixellated gas tracker is that the direction of the Compton recoil electron or the low-energy nuclear recoil can be reconstructed far more accurately than in any other detection medium.

One of the most exciting future applications of GEM and Micromegas devices with CMOS multi-pixel readout could be position sensitive single photon detection. The excellent spatial and time resolution, ambiguity-free reconstruction of multi-photon events and non-negligible single-electron sensitivity make them a suitable candidate for fast gas photo-multipliers.

A key point that has to be solved to allow using CMOS pixel readout of MPGDs in high-energy physics is the production of large area detectors. Recent progress in the development of edgeless silicon detectors [61] and the pos- 
sibility to bring power and I/O connections through the back of the CMOS chip using the "through-wafer vias" technology $[62,63]$ may ultimately lead to the development of chips which are 4-side buttable. Properly integrated into large systems, the multi-pixel anode readout of micro-pattern gas detectors may represent an invaluable tool for the next generation of particle-physics experiments. However, a major $R \& D$ effort will be required in the future to fully exploit this potential.

\subsection{GEM with a VLSI Pixel ASIC for X-Ray Astronomy}

A GEM detector coupled to a VLSI analog pixel chip comprising a pixellated charge collecting electrode and readout electronics, can bring great improvement in sensitivity, at least 2 orders of magnitude, compared to traditional $X$-ray polarimeters (based on Bragg crystals or Compton scattering) [45]. The novel device allows to reconstruct individual photoelectron tracks with a length as short as a few hundred microns; the total charge collected in the pixels is proportional to the photon energy. The degree of $X$-ray polarization is computed from the distribution of reconstructed track angles since the photoelectron is emitted mainly in the direction of the photon electric field. Three ASIC generations of increased complexity and size, reduced pitch and improved functionality have recently been designed and built [46]-[48]. The third ASIC version, realized in $0.18 \mu \mathrm{m}$ CMOS technology, includes a self-triggering capability and has 105600 hexagonal pixels with $50 \mu \mathrm{m}$ pitch, corresponding to an active area of $15 \times 15 \mathrm{~mm}^{2}$. A GEM coupled to such a CMOS pixel array is able to simultaneously produce high-resolution images $(50 \mu \mathrm{m})$ and allow moderate spectroscopy (15\% FWHM at $6 \mathrm{keV}$ ) as well as fast timing (30 ns) in the $2-10 \mathrm{keV} X$-ray energy range. At the focal plane of the large-area mirror of the XEUS telescope, a single $15 \times 15 \mathrm{~mm}^{2}$ ASIC will cover a field of view of 5 arcmin, large enough to image extended objects like the Crab Nebula. Because of the high detector sensitivity, the polarization of Active Galactic Nuclei down to the few-\% level can be measured for $1 \mathrm{mCrab}$ sources in one day [48]. A sealed gas pixel detector for $X$-ray astronomy is currently under development [49].

Recently, a UV photo-detector based on a semitransparent CsI photocathode followed by a fine-pitch GEM foil matching the pitch of a pixel ASIC $(50 \mu \mathrm{m})$ has shown excellent imaging capabilities [64]. The photoelectron emitted from the CsI layer drifts into a single GEM hole and initiates an avalanche, which is then collected on the pixel CMOS analog chip. Due to the high granularity and large $S / N$ of the read-out system, the "center of gravity" of the single electron avalanche corresponds to the center of GEM hole. Accumulating thousands of such events produces the "self-portrait" of the GEM amplification structure shown in Fig. 6 (a). The peaks, corresponding to GEM holes, are well resolved 
a)

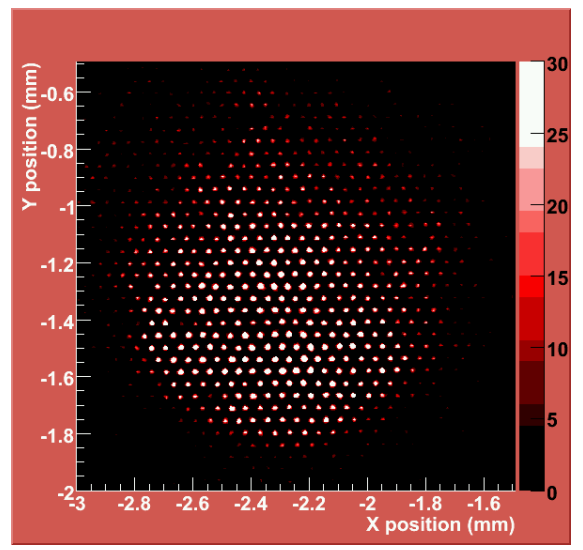

b)

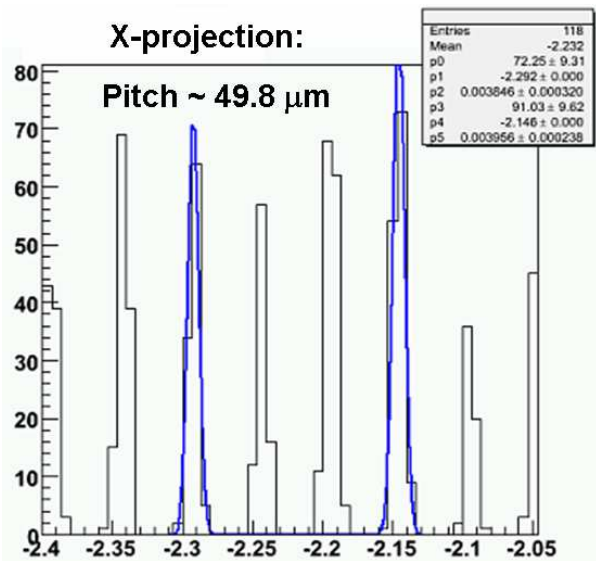

Fig. 6. a) "Self-portrait" of the GEM amplification structure obtained with a CMOS analog pixel chip. b) A horizontal cut through the 2D image of barycenter positions ("centre of gravity" of the single electron avalanche). The peak-to-peak distance corresponds to the GEM pitch. The gaussian fit yields $\sim 4 \mu \mathrm{m}$ (rms) width [64].

in the barycenter distribution in Fig. 6 (b) allowing to achieve a superior single-electron avalanche reconstruction accuracy of $4 \mu \mathrm{m}$ (rms). Thanks to the very low pixel capacitance at the preamplifier input (noise $\sim 50 e^{-} \mathrm{ENC}$ ), the detector has significant sensitivity to a single primary electron even at a gas gain of a few thousand. The position resolution of the device is currently limited by the $50 \mu \mathrm{m}$ GEM pitch. The symmetric shape of a single-electron charge cloud at the readout plane demonstrates that the spatial resolution is not degraded by the avalanche spread inside the GEM and is independent of the direction of entrance of the electron into the hole.

\subsection{Micromegas Readout with Medipix2 / TimePix CMOS Chips}

Initial 'proof-of-principal' studies using Micromegas foils equipped with the Medipix2 chip provided 2D images of minimum ionizing track clusters [51,52]. The single (primary) electron efficiency was estimated to be around $90 \%$; the number of clusters in a $\mathrm{He} / \mathrm{iC}_{4} \mathrm{H}_{10}$ (80:20) mixture was found to agree within $15 \%$ with simulation. The Timepix chip was also demonstrated to function perfectly with Micromegas; 3D track images from radioactive sources and cosmic rays have been observed in the NIKHEF setup (see Fig. 7a) [65]. Prior to operation the Timepix chip was covered with a highly resistive $4 \mu \mathrm{m}$ layer of amorphous silicon as protection against sparks and discharges.

An attractive solution for the construction of MPGDs with pixel anode readout is the integration of the Micromegas amplification and CMOS chip by means of the 'wafer post-processing' technique [66]. With this technology, the structure of a thin $(1 \mu \mathrm{m})$ aluminum grid is fabricated on top of an array of insulating (SU-8) pillars of typically $50 \mu \mathrm{m}$ height standing above the CMOS chip and forming an integrated readout of the gaseous detector (InGrid). The sub- $\mu m$ 
a)

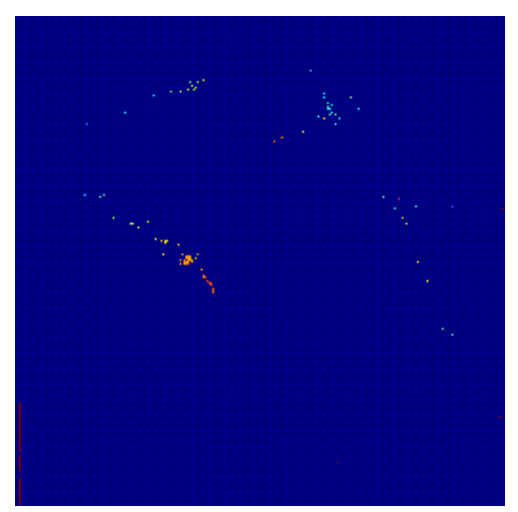

b)

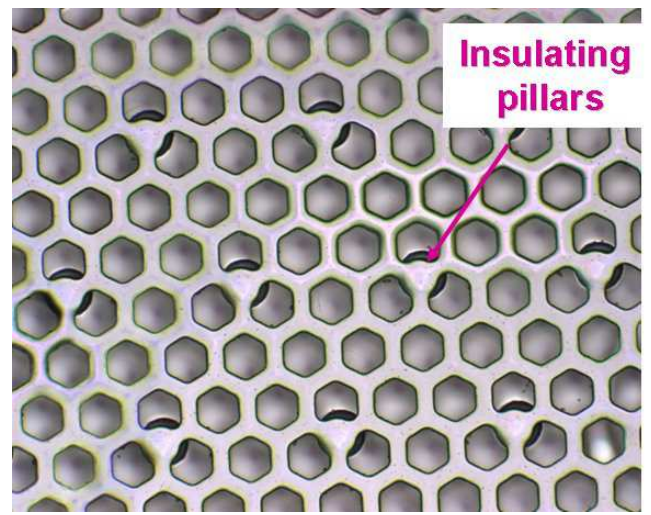

Fig. 7. a) Cosmic rays recorded with a Micromegas/Timepix detector ("TOT" mode); the color is a measure of the arrival time of the electrons [65]. b) Top view of InGrid structure with hexagonal holes (pitch $60 \mu \mathrm{m}$ ). The insulating pillars (SU-8 epoxy) are centered between the grid holes.

precision of the grid dimensions and avalanche gap size results in a uniform gas gain; the grid hole size, pitch and pattern can be easily adapted to match the geometry of any pixel readout chip. The 'wafer post-processing' technology can also be used if the readout CMOS matrix does not exactly match the required detector granularity. "Through-wafer vias" connections with variable re-routing lines allow to use detector elements with slightly smaller readout chips and space left over for external connections [62].

\subsection{Triple-GEM Readout with Medipix2 / Timepix CMOS Chips}

The triple-GEM detector with a Medipix2 chip has been initially studied in the Freiburg University with ${ }^{55} \mathrm{Fe} X$-Rays and ${ }^{106} \mathrm{Ru}$ electrons [53]. Stable operation at the gas gain of up to several $10^{5}$ has been achieved with $\mathrm{Ar}(\mathrm{He}) / \mathrm{CO}_{2}$ (70:30) mixtures. The device allows to perform moderate energy spectroscopy measurements (20\% FWHM at $5.9 \mathrm{keV} X$-rays) using only digital readout and two discriminator thresholds. A sample of ${ }^{106} \mathrm{Ru} \beta^{-}$-tracks was collected and the point resolution was evaluated using various methods and taking into account the multiple scattering contribution of electrons with a few MeV. A spatial resolution of $\sim 50 \mu \mathrm{m}$ (averaged over tracks in the $6 \mathrm{~mm}$ drift gap) has been achieved, based on the binary centroid determination of the charge clouds.

Later, the GEM/Medipix2 detector was exposed to a $5 \mathrm{GeV}$ electron beam at DESY (see Fig. 8a). The dependence of the spatial resolution on the drift length ( $y$-coordinate of the track inside the $6 \mathrm{~mm}$ GEM drift volume) was measured using external Si-telescope planes [67]. The deviations of the "center of gravity" of individual cluster avalanches from the straight-line fit can be 
a)

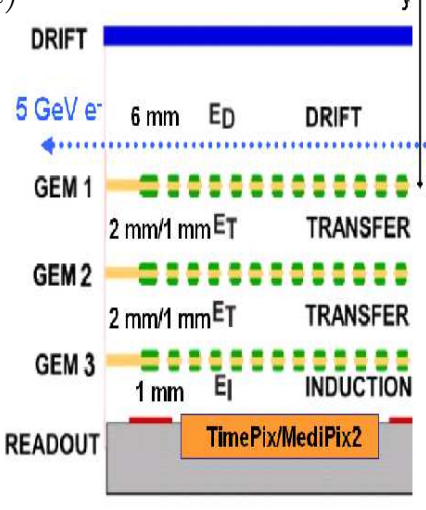

b)

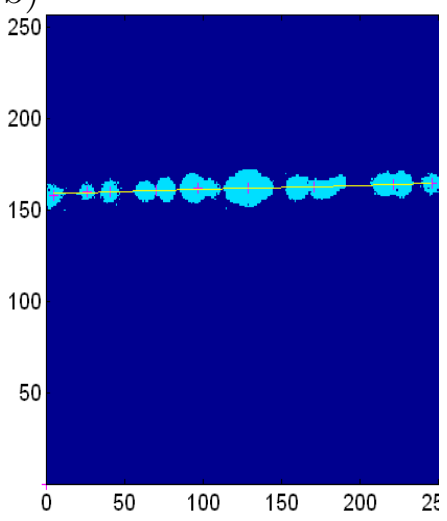

c)

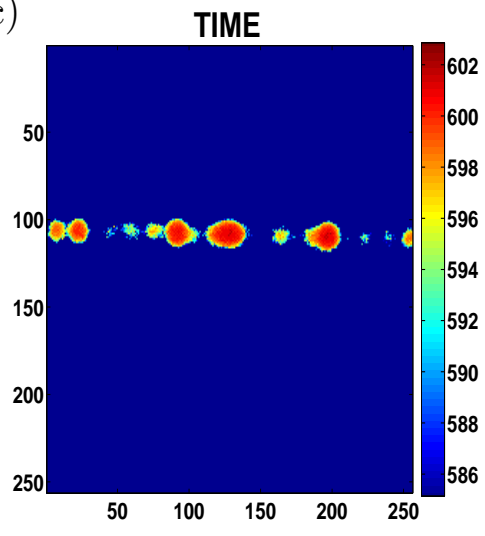

Fig. 8. a) A schematic drawing of the GEM/Medipix2 (Timepix) detector with an electron beam crossing the drift volume. b) and c) Images of $5 \mathrm{GeV}$ electron tracks in $\mathrm{Ar} / \mathrm{CO}_{2}$ (70:30) recorded with Medipix2 and Timepix ("TIME" mode) chips. A straight-line fit to the cluster centers is shown. The $x, y$-axes represent the chip's sensitive area, arranged as a square matrix of $256 \times 256$ pixels of $55 \times 55 \mu^{2}$ size.

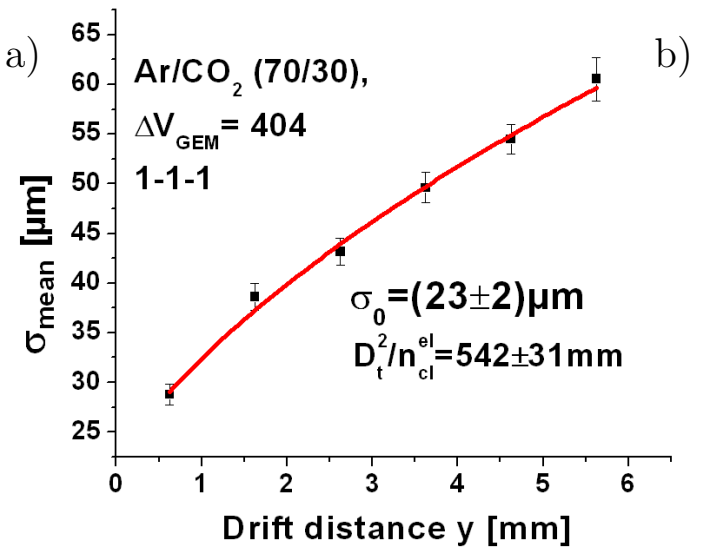

\begin{tabular}{|c|c|c|c|}
\hline $\begin{array}{c}\text { Gas } \\
\text { mixture }\end{array}$ & $\begin{array}{c}\text { Detector } \\
\text { configu- } \\
\text { ration }\end{array}$ & $\begin{array}{c}\text { MEDIPIX2 } \\
\sigma_{0}(\mu \mathrm{m})\end{array}$ & $\begin{array}{c}\text { TIMEPIX } \\
\sigma_{0}(\mu \mathrm{m})\end{array}$ \\
\hline $\mathrm{Ar} / \mathrm{CO}_{2}$ & $2-2-1$ & -- & $24 \pm 2$ \\
\hline $\mathrm{Ar} / \mathrm{CO}_{2}$ & $1-1-1$ & $23 \pm 2$ & - \\
\hline $\mathrm{He} / \mathrm{CO}_{2}$ & $2-2-1$ & $32 \pm 2$ & $30 \pm 2$ \\
\hline $\mathrm{He} / \mathrm{CO}_{2}$ & $1-1-1$ & $29 \pm 4$ & -- \\
\hline
\end{tabular}

Fig. 9. a) Spatial resolution $\left(\sigma_{\text {mean }}\right)$ as a function of drift distance in an $\mathrm{Ar} / \mathrm{CO}_{2}$ (70:30) mixture in a triple-GEM/Medipix2 detector. b) Summary of intrinsic resolution studies $\left(\sigma_{0}\right)$ for different GEM/Medipix2 and GEM/Timepix stack configurations in $\mathrm{Ar}(\mathrm{He}) / \mathrm{CO}_{2}(70: 30)$ mixtures.

parameterized as:

$$
\sigma_{\text {mean }}^{2}(y)=\sigma_{0}^{2}+\sigma_{d i f}^{2}(y)=\sigma_{0}^{2}+\frac{D_{t}^{2} \cdot y}{n_{c l}^{e l}},
$$

where, $\sigma_{0}$ is the GEM "defocussing" term (depends on diffusion of amplified electrons in the triple-GEM structure and on the GEM hole/pitch size), $D_{t}$ is a transverse diffusion coefficient, $n_{c l}^{e l}$ is the effective number of primary electrons per cluster contributing to resolution, and $y$ is the drift distance from the surface of the first GEM. Diffusion of primary ionization clusters as a function of the drift distance was clearly observed, as shown in Fig. 9a. The intrinsic spatial resolution, extrapolated to zero drift length $(y=0)$, of $\sigma_{0} \approx 23-32 \mu \mathrm{m}$ was measured for different GEM/Medipix2 stack configurations (see Fig. 9b). 
The triple-GEM device has been also operated with Timepix readout (both in "TIME" and "TOT" modes) in the same setup at the DESY beam. Fig. 8c shows an electron track recorded in the "TIME" mode; the color denotes the arrival time of electrons in a pixel. Similar spatial resolution of $\sigma_{0} \approx 24-30 \mu \mathrm{m}$ was achieved with the Timepix chip for tracks close to the surface of the first GEM (see Fig. 9b). It should also be noted that the measured point resolution $\left(\sigma_{0} \sim 25 \mu \mathrm{m}\right)$ is currently affected by the finite distance between GEM holes $(140 \mu \mathrm{m})$; a better resolution can be achieved with GEMs of smaller pitch. These results demonstrate that the CMOS readout of MPGDs meets the requirements for tracking detectors in the next generation of high-energy colliders.

\section{Radiation Hardness of Gaseous Detectors}

Aging phenomena constitute one of the most complex and serious potential problems which could limit, or severely impair, the use of gaseous detectors in unprecedented harsh radiation environments. The "classical aging effects" are the result of chemical reactions occurring in avalanche plasma in wire chambers, which lead to formation of deposits - conductive or insulating - on the electrode surfaces and manifest themselves by a decrease of the gas gain, excessive currents, sparking and self-sustained discharges. Over the last decade considerable progress has been made in understanding the basic rules for the construction and operation of gaseous detectors. Long life in the high-intensity environments of the LHC-era demands not only extraordinary radiation hardness of construction materials and gas mixtures but also very specific and appropriate assembly procedures and quality checks during detector construction and testing. Only a limited choice of aging-resistant gases can be successfully used at high-luminosity colliders: noble gases, $\mathrm{CF}_{4}, \mathrm{CO}_{2}, \mathrm{O}_{2}, \mathrm{H}_{2} \mathrm{O}$. Hydrocarbons are not trustable for long-term high-rate experiments.

A detailed discussion of aging phenomena is beyond the scope of this paper. The achievements of past $R \& D$ projects are summarized in $[68,69,70,71]$, the most recent developments in radiation hardness research with state-of-the-art gaseous detectors are reviewed in $[72,73,74]$. Detailed studies of micro-pattern GEM and Micromegas concepts have revealed that they might be even less vulnerable to radiation-induced performance degradation in harsh radiation environments than standard silicon microstrip detectors. Recently, reliable operation has been established for GEM detectors coupled to VLSI analog ASICs or Medipix2 CMOS chips; no single pixel chip has been destroyed after several months of running $[47,53]$. More than twenty years of intensive research aimed at matching the needs of high-luminosity colliders have demonstrated that if properly designed and constructed, gaseous detectors can be robust and stable in the presence of high rates and heavily ionizing particles. 


\section{Micro-Pattern Gas Detector Applications}

The performance, robustness and radiation hardness of MPGDs have encouraged their applications in many other fields; a short summary of the most recent results is given below.

\subsection{Gaseous Photomultipliers}

Large-area RICH gaseous detectors with a thin photosensitive CsI-layer deposited on the cathodes of a MWPC are currently employed for particle identification in many high-energy physics experiments [75,76]. In recent years there has been considerable progress in the field of photon detection by combining MPGDs with semi-transparent or reflective CsI photocathodes (PC) to localize single photoelectrons [77]. These detectors offer high gain even in noble gases, sub-nanosecond time response, and excellent localization properties and are able to operate in high magnetic fields and at cryogenic temperatures. Using a triple-GEM detector with hexagonal readout, a position accuracy of $55 \mu \mathrm{m}$ and a two-photon separation of around $1 \mathrm{~mm}$ have been achieved [78]. A Micromegas filled with a $\mathrm{He} / \mathrm{iC}_{4} \mathrm{H}_{10}$ mixture at atmospheric pressure allows to achieve a time resolution of $\sim 700 \mathrm{ps}$ for single photoelectrons [13]. The hole-type gaseous structures: GEMs and Cappillary Plates coupled to CsI-PC can operate stably down to $80 \mathrm{~K}[79,80,81]$.

The operation of MPGD-based photomultipliers in $C F_{4}$ with CsI-PC could form the basis of new-generation windowless Cherenkov detectors where both the radiator and the photosensor operate in the same gas. Exploiting this scheme a Hadron Blind Detector (originally proposed for a Parallel Plate Avalanche Chamber $[82,83,84])$ has been recently developed and constructed using a triple-GEM amplification system as part of the upgrade program for the PHENIX experiment at RHIC [85,86]. Hadron blindness is achieved by reversing the direction of the drift field $E_{D}$, therefore pushing primary ionization produced by charged particles towards the mesh. In this configuration photoelectrons released from the CsI surface are still efficiently collected into the GEM holes and multiplied. The avalanche confinement within the GEM holes strongly reduces photon-mediated secondary processes in $C F_{4}$ (CsI is sensitive to the $C F_{4}$ scintillation peak at $170 \mathrm{~nm}$ ) [87].

The success of GEMs and glass capillary plates triggered the development of coarse and more robust structures, "optimized GEMs" [88,89] followed by think-GEM (THGEM) [90,91] gaseous multipliers made of standard PCB perforated with sub-millimeter diameter holes etched at their rims. Effective gas amplification factors of $10^{5}$ and $10^{7}$ and fast pulses of a few nanoseconds rise- 
time were reached in single and cascaded double-THGEM elements. Stable operation with high single-photoelectron detection efficiency was recorded at fluxes exceeding $\mathrm{MHz} / \mathrm{mm}^{2}$. A novel spark-protected version of a thick GEM with electrodes made of resistive kapton (RETGEM) has been recently developed [92]. At low counting rates the detector operates as an conventional THGEM with metallic electrodes while at high intensities and in case of discharges the behavior is similar to that of a resistive-plate chamber. Recent studies of photosensitive RETGEMs with CsI deposited directly on the dielectric kapton (without metallic substrate) have shown a rather high quantum efficiency (34\% at $120 \mathrm{~nm}$ ) [93]. Application of the THGEM and RETGEM concepts to the RICH technology promises to enhance its particle identification capabilities.

\subsection{Optical Readout of GEM-based Detectors}

Scintillation light emitted during the development of electron avalanches can be effectively used for the optical readout of gaseous detectors. The luminescence processes in GEMs read out by high-resolution CCDs or by PMTs have been used for imaging X-rays, $\alpha$-particles and thermal neutrons [94]. Particular attention has been given to mixtures emitting in the visible and near-infrared regions (from $400 \mathrm{~nm}$ to $1000 \mathrm{~nm}$ ), the sensitivity region of the CCD. One of the most promising candidates having adequate scintillation spectra is the $A r / C F_{4}$ (95:5) mixture, where $\sim 0.7$ photons above $400 \mathrm{~nm}$ are emitted per secondary electron [95]. The possibility to use a GEM-based scintillation readout of a TPC as a 3D tracking detector has been reported in [96]. The double GEM filled with $\mathrm{Ar} / \mathrm{CF}_{4}$ (95:5) and coupled to a $2 \times 2$ matrix of $39-\mathrm{mm}$ diameter PMTs, was tested with ${ }^{241} \mathrm{Am} 5.48 \mathrm{MeV} \alpha$-particles, emitted under various track angles $(\theta)$ with respect to the drift plane. The arrival positions of the primary electrons in the GEM plane were determined from the center of gravity of the PMT light pulses, analyzed in 9 equal time intervals as shown in Fig. 10a. A typical track angle resolution of $2^{\circ}(\sigma)$ at $\theta \sim 45^{\circ}$ and a spatial resolution of better than $1 \mathrm{~mm}(\mathrm{FWHM})$ were achieved.

Another application of the GEM scintillation detector is radiation therapy, which demands new online beam-monitoring systems with $\sim 1 \mathrm{~mm}$ position resolution and 3-D dosimetry of delivered doses with an accuracy of $\sim 5 \%$. The light yield of a Lanex scintillating screen coupled to a CCD camera, which is currently used for the quality control of clinical beams, can underestimate the dose by $\sim 30 \%$ over the last part of proton track (the Bragg peak) with respect to the reference ionization chamber. A double GEM detector coupled to a CCD camera has been developed to detect photons emitted by $A r / C F_{4}$ (96:4) excited molecules; the total charge extracted from the GEM holes was also measured [97]. The intensity of the measured light pattern gives directly 
a)

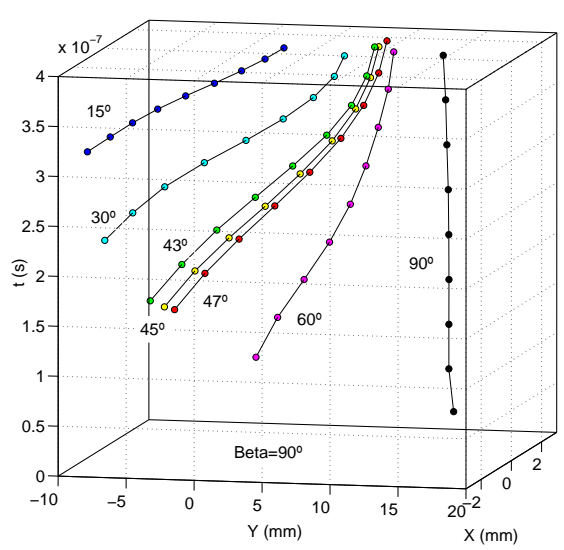

b)

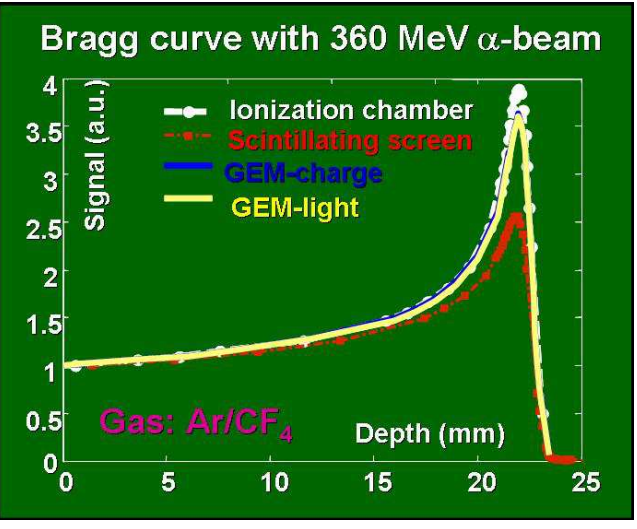

Fig. 10. a) 3D track reconstruction in the GEM TPC using optical readout. Individual track PMT pulses were analyzed in 9 time samples. Each dot corresponds to the center of gravity of one sample along the track. b) Comparison between Bragg curves measured with the reference ionization chamber, the GEM detector (charge and light signals), and the Lanex screen from [98].

a 2-D distribution of the energy deposited in the sensitive GEM volume by primary electrons, integrated over all the beam time. The $3^{\text {rd }}$ dimension can be obtained by placing different thicknesses of tissue-equivalent material in front of the detector. With a $360 \mathrm{MeV} \alpha$-beam the integrated light yield was found to be linearly proportional to the total charge extracted from the holes of a $2^{\text {nd }}$ GEM, and the scintillating GEM light signal at the Bragg peak depth was only $4 \%$ smaller than that of the reference ionization chamber (see Fig. 10b) [98]. Consequently, the scintillating GEM with CCD readout may become a feasible substitute for the Lanex screen, especially at high ionization densities of alpha or carbon-ion beams.

\subsection{Micromegas for Neutron Detection and Low Background Experiments}

There are many applications of the Micromegas concept in the neutron detection domain, which include neutron beam diagnostics [99], inertial fusion experiments [100], thermal neutron tomography [101] and a novel compact sealed Picollo-Micromegas detector designed to provide in-core measurements of the neutron flux and energy (from thermal to several $\mathrm{MeV}$ ) in nuclear reactors [102]. Neutrons can be converted into charged particles to detect ionization in Micromegas by two means: either using the detector gas filling or a target with appropriate deposition in its entrance window. Micromegas detectors are also used in searching for solar axions (CAST) [103] and under development for low energy neutrino experiments (HELLAZ, NOSTOS) [104,105], including measurements of neutrino oscillations and the neutrino magnetic moment. In particular, in the CAST experiment at CERN, the expected signal comes from solar axion conversions into low-energy photons of 1-10 keV energy. A Micromegas detector with high granularity anode elements can largely 
reduce the background event rate down to $5 \times 10^{-5} \mathrm{keV}^{-1} \mathrm{~cm}^{-2} \mathrm{~s}^{-1}$, exploiting its stability, good energy and spatial resolution [106].

\section{$7 \quad$ Summary and Outlook}

Today's LHC gaseous detectors have opened a new era of state-of-the-art technologies and are the benchmarks for developments beyond the LHC. Advances in photo-lithography and micro-processing techniques in the chip industry during the past decade triggered a major transition in the field of gas detectors from wire structures to micro-pattern devices. The GEM and Micromegas detectors became a wide-spread tool for high-rate tracking over large sensitive areas, precision reconstruction of charged particles in the TPC, $X$-ray, UV and visible photon detection and neutron spectroscopy. Modern, sensitive and low-noise electronics will enlarge the range of applications. As a part of this development, a micro-pattern gas detector with finely segmented CMOS readout can be used as an ultra-high-precision "electronic bubble chamber" (see Fig. 11), opening a window to new physics for many applications, especially for low-energy charged-particle and photon detection.

a)

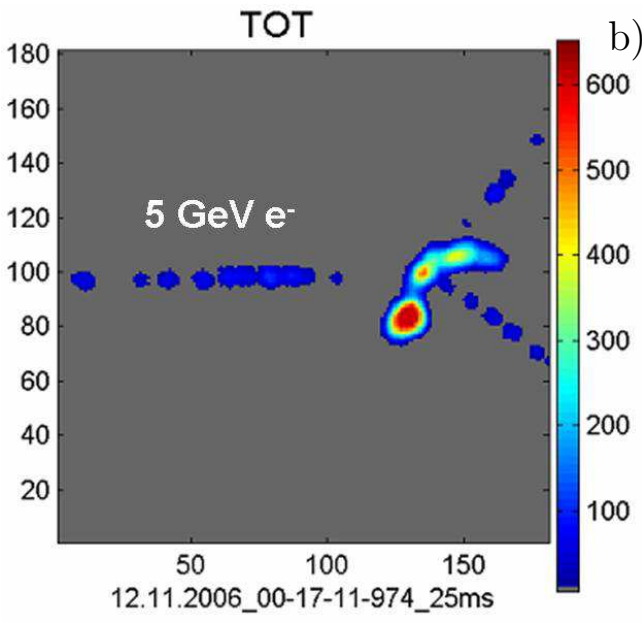

TIME

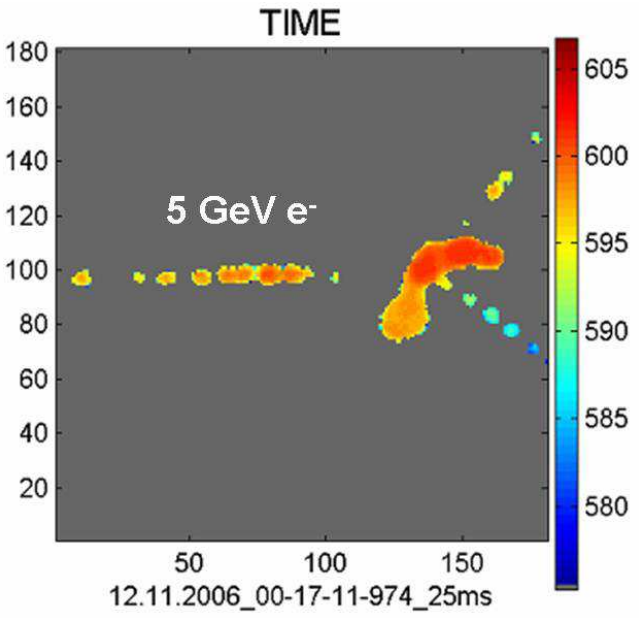

Fig. 11. Inelastic interaction of the $5 \mathrm{GeV}$ electron at the DESY testbeam recorded with triple-GEM detector readout with a TimePix CMOS chip operated in mixed mode: every second pixel is operated in the "TOT" or "TIME" modes, respectively, in a "chess board" fashion. The $x, y$-axes represent the chip's sensitive area, obtained by mapping the original data (matrix of $256 \times 256$ pixels of $55 \mu \mathrm{m}$ pitch) onto a $181 \times 181$ pixels matrix with a pitch of $78 \mu \mathrm{m}$. The color is a measure of time-over-threshold and drift time information, respectively. 


\section{Acknoledgements}

I would like to thank M. Jeitler, M. Krammer, W. Mitaroff and M. Regler for providing a wonderful cultural and scientific environment during the Vienna Conference. I would like to express my gratitude to A. Bamberger, R. Bellazzini, P. Colas, K. Desch, I. Giomataris, E. Heijne, V. Peskov and F. Sauli for many helpful suggestions, stimulating discussions and careful reading of this

manuscript. I would like to thank U. Bratzler for reading and correcting this manuscript and U. Renz for helping in the preparation of the paper.

\section{References}

[1] https://twiki.cern.ch/twiki/bin/view/Atlas/MuonSpectrometer

[2] J. Dubbert, paper presented at this conference.

[3] T. Virdee, Physics Reports, 403-404(2004), 401.

[4] D. Froidevaux, Annu. Rev. Nucl. Part. Sci., 56(2006), 375.

[5] F. Sauli, A. Sharma, Ann. Rev. Nucl. Part. Sci. 49(1999) 341.

[6] A. Oed, Nucl. Instr. Meth., A263(1988), 351.

[7] Y. Bagaturia et al., Nucl. Instr. Meth., A490(2002), 223.

[8] J. Benlloch et al., IEEE Trans. Nucl.Sci. 45(1998), 234.

[9] I. Giomataris, Nucl. Instr. Meth., A419(1998), 239.

[10] A. Bressan et al., Nucl. Instr. Meth., A425(1999), 262.

[11] J. Derre et al., Nucl. Instr. Meth., A459(2001), 523.

[12] A. Breskin et al., Nucl. Instr. Meth., A483(2002), 670.

[13] Y. Derre et al., Nucl. Instr. Meth., A449(2000), 314.

[14] F. Sauli, Nucl. Instr. Meth., A386(1997), 531.

[15] S. Bachmann et al., Nucl. Instr. Meth., A470(2001), 548.

[16] S. Bachmann et al., Nucl. Instr. Meth., A479(2002), 294.

[17] http://gdd.web.cern.ch/GDD/

[18] L. Ropelewski, paper presented at this conference.

[19] G. Bencivenni, paper presented at this conference.

[20] A. Bondar et al., Nucl. Instr. Meth., A556(2006), 495. 
[21] U. Becker et al., Nucl. Instr. Meth., A556(2006), 527.

[22] B. Azmoun et al., 2006 IEEE NSS/MIC Conference Record.

[23] Y. Giomataris, Nucl. Instr. Meth., A376(1996), 29.

[24] A. Delbart et al., Nucl. Instr. Meth., A461(2001), 84.

[25] I. Giomataris et al., Nucl. Instr. Meth., A560(2006), 405.

[26] J. Bouchez et al., "Bulk Micromegas detectors for large TPC applications", submitted to Nucl. Instr. Meth., A

[27] A. Sarrat, paper presented at this conference.

[28] I. Giomataris, private communations

[29] B. Ketzer et al., Nucl. Instr. Meth., A535(2004), 314.

[30] C. Bernet et al., Nucl. Instr. Meth., A536(2005), 61.

[31] B. Ketzer, paper presented at this conference.

[32] G. Barouch et al., Nucl. Instr. Meth., A423(1999), 32.

[33] M. Alfonsi et al., IEEE Trans. Nucl.Sci. 51(5)(2004), 2135.

[34] S. Lami et al., e-Print:physics/0611178.

[35] D. Nygren el at., Proposal PEP No 4, Appendix A 6.

[36] D. Antonczyk el at., Nucl. Instr. Meth., A565(2006), 551.

[37] U. Frankenfeld, paper presented at this Conference.

[38] T. Meyer, Proc. of the 42nd Workshop of the INFN ELOISATRON Project, "Innovative Detectors For Super-Colliders, Erice, Italy, Sep.28-Oct.4 (2003).

[39] LC-TPC

Collaboration, https://wiki.lepp.cornell.edu/ilc/bin/view/Public/WWS/

[40] M.E. Janssen, 2006 IEEE NSS/MIC Conference Record.

[41] M. Dixit et al., e-Print physics/0703243, paper presented at this Conference.

[42] M. Dixit, A. Rankin, Nucl. Instr. Meth., A566(2006), 281.

[43] F. Sauli et al., Nucl. Instr. Meth., A560(2006), 269.

[44] S.X. Oda et al., Nucl. Instr. Meth., A566(2006), 312.

[45] E. Costa et al., Nature, Vol. 411(2001), 662.

[46] R. Bellazzini et al., Nucl. Instr. Meth., A535(2004), 477.

[47] R. Bellazzini et al., Nucl. Instr. Meth., A560(2006), 425.

[48] R. Bellazzini et al., Nucl. Instr. Meth., A566(2006), 552. 
[49] R. Bellazzini et al., e-Print: astro-ph/0604114.

[50] X. Llopart, M. Campbell, IEEE Trans. Nucl. Sci., 49(5)(2002), 2279

[51] P. Colas et al., Nucl. Instr. Meth., A535(2004), 506.

[52] M. Campbell et al., Nucl. Instr. Meth., A540(2005), 295.

[53] A. Bamberger et al., Nucl. Instr. Meth., A573(2007), 361.

[54] EUDET: An integrated infrastructure initiative funded under the $6^{\text {th }}$ EU framework, see http://www.eudet.org

[55] X. Llopart et al., paper presented at this Conference.

[56] http://hausch.home.cern.ch/hausch/MediPix.html

[57] H. Nishimura et al., Proceedings of the 2006 IEEE NSS/MIC conference.

[58] K. Hattori et al., paper presented at this Conference.

[59] H. Sekiya et al., Nucl. Instr. Meth., A573(2007), 204.

[60] K. Miuchi et al., e-Print: physics/0701181.

[61] C. Da Via et al., Nucl. Instr. Meth., A509(2003), 86.

[62] E. Heijne, Nucl. Instr. Meth., A541(2005), 274.

[63] K. Takahashi et al., Microelectronics Realibility, 43(2003), 1267.

[64] R. Bellazzini et al., paper presented at this Conference.

[65] J. Timmermans et al., paper presented at this Conference.

[66] M. Chefdeville et al., Nucl. Instr. Meth., A556(2006), 490.

[67] A. Bamberger et al., paper presented at this Conference.

[68] J. Va'vra, Nucl. Instr. Meth., A252(1986), 547.

[69] J. Kadyk, Nucl. Instr. Meth., A300(1991), 436.

[70] R. Bouclier, Nucl. Instr. Meth., A381(1996), 289.

[71] Proceedings of the Workshop on Radiation Damage to Wire Chambers, LBL21170, (1986).

[72] Proceedings of the International workshop on Aging Phenomena in Gaseous Detectors, Nucl. Instr. and Methods A515, (2003).

[73] M. Titov et al, e-Print: hep-ex/0204005, IEEE Trans. Nucl. Sci. NS49(4),1609 (2002).

[74] M. Titov, e-Print: physics/0403055; Proc. of the 42nd Workshop of the INFN ELOISATRON Project, "Innovative Detectors For Super-Colliders, Erice, Italy, Sep.28-Oct.4 (2003). 
[75] F. Piuz, Nucl. Instr. Meth., A502(2003), 76.

[76] E. Nappi, Nucl. Instr. Meth., A471(2003), 18.

[77] R. Chechik et al., Nucl. Instr. Meth., A502(2003), 195.

[78] F. Sauli, Nucl. Instr. Meth., A553(2005), 18.

[79] L. Periale et al., Nucl. Instr. Meth., A535(2004), 517.

[80] L. Periale at al., IEEE Trans. Nucl. Sci., 52(4)(2005), 927.

[81] D. Pavlyuchenko et al., paper presented at this Conference.

[82] Y. Giomataris, G. Charpak, Nucl. Instr. Meth., A310(1991), 585.

[83] M. Chen et al., Nucl. Instr. Meth., A346(1994), 120.

[84] I. Giomataris, Proc. of the SNIC Symposium, Stanford, California, Apr.3-6 (2006).

[85] Z. Fraenkel et al., Nucl. Instr. Meth., A546(2005), 466.

[86] C. Woody et al., Proceedings of the 2006 IEEE NSS/MIC conference.

[87] A. Breskin et al., Nucl. Instr. Meth., A483(2002), 670.

[88] L. Periale at al., Nucl. Instr. Meth., A478(2002), 377.

[89] L. Periale at al., IEEE Trans. Nucl. Sci., 50(4)(2003), 809.

[90] R. Chechik et al., Nucl. Instr. Meth., A535(2004), 303.

[91] C. Shalem et al., Nucl. Instr. Meth., A558(2006), 475.

[92] R. Oliveira et al, e-Print: physics/0701154.

[93] A. Di Mauro et al, e-Print: arXiv:0706.0102, paper presented at this Conference.

[94] F.A.F Fraga et al., Nucl. Instr. Meth., A513(2003), 379.

[95] M.M.F.R. Fraga et al., Nucl. Instr. Meth., A504(2003), 88.

[96] S.T.G. Fetal et al., paper presented at this Conference.

[97] S. Fetal et al., Nucl. Instr. Meth., A513(2003), 42.

[98] F. Seravalli et al., First Results of a Scintillating GEM Detector for 2D dosimetry in $\alpha$-beam, submitted to IEEE Trans. Nucl. Sci. (2007)

[99] J. Pancin et al., Nucl. Instr. Meth., A524(2004), 102.

[100] M. Houry et al., Nucl. Instr. Meth., A557(2006), 648.

[101] S. Andriamonje et al., 2004 IEEE NSS/MIC Conference Record.

[102] S. Andriamonje et al., Nucl. Instr. Meth., A562(2006), 755. 
[103] P. Abbon et al., 2006 IEEE NSS/MIC Conference Record.

[104] P. Gorodetsky et al., Nucl. Phys. Proc. Suppl., 87(2000), 506.

[105] I. Giomataris, J.D. Vergados, Nucl. Instr. Meth., A530(2004), 330.

[106] E. Ferrer Ribas et al., paper presented at this Conference. 